\title{
Disturbing and distressing: a mixed methods study on the psychological impact of end of life care on junior doctors
}

\author{
Hannah Linane ${ }^{1}$ (1) $\cdot$ Fergal Connolly $^{2,3} \cdot$ Lyle McVicker $^{2,3} \cdot$ Sharon Beatty $^{1} \cdot$ Orla Mongan $^{2,3} \cdot$ Eileen Mannion ${ }^{1}$. \\ Dympna Waldron ${ }^{1,2} \cdot$ Dara Byrne ${ }^{2,3}$
}

Received: 28 June 2018 / Accepted: 9 August 2018 / Published online: 24 August 2018

(C) Royal Academy of Medicine in Ireland 2018

\begin{abstract}
Background The Irish Medical Council has identified gaps in knowledge and communications skills of new-entrant doctors in legal, ethical and practical aspects of end of life care.

Aims To determine the frequency with which junior doctors deal with end of life care and patient death and to evaluate the impact this has on their psychological wellbeing.

Design A questionnaire was distributed to junior doctors to determine the frequency with which the participants had managed a patient at end of life. An abbreviated Posttraumatic Stress Disorder Checklist-Civilian version was used to evaluate psychological distress. Critical incident technique interviews explored the challenges of caring for patients at end of life.

Setting/participants A total of 110 junior doctors in two teaching hospitals in Ireland completed the questionnaire: 39 (35.5\%) interns and 71 (64.5\%) senior house officers. In addition, 31 interviews were carried out with interns, senior house officers and registrars.

Results The majority $(81.8 \%$ ) had pronounced a death with $39.4 \%$ of senior house officers doing so more than 10 times. Three quarters (75.5\%) had discussed end of life with a patient's family. Of the 110 respondents that completed the posttraumatic stress disorder checklist, $11.8 \%$ screened positively for posttraumatic stress disorder. Challenges identified at interview included lack of knowledge and preparedness, difficulty communicating with family members, a lack of support and a feeling of failure.

Conclusions Junior doctors are regularly carrying out tasks related to end of life care, resulting in high levels of psychological distress. Further training and a change in culture are required.
\end{abstract}

Keywords Death $\cdot$ Education $\cdot$ Palliative care $\cdot$ Physicians $\cdot$ Psychological $\cdot$ Stress

\section{Key statements}

- What is already known about the topic There are gaps in knowledge and communications skills of new-entrant doctors around the legal, ethical and practical aspects of end of life care.

- What this paper adds Junior doctors are reporting a high level of psychological distress as a result of managing patient death and end of life care.

- Implications for practice, theory or policy Further training is required to improve preparedness for patient death and end of life care.

Junior doctors require support when dealing with distressing clinical situations.

Hannah Linane

hannahlinane@gmail.com

1 Department of Palliative Medicine, University Hospital Galway, Galway, Ireland

2 National University of Ireland, Galway, Ireland

3 Irish Centre for Applied Patient Safety and Simulation, Galway, Ireland

\section{Introduction}

Acquiring the skills and attitudes required to be competent and comfortable with end of life care and death is challenging and poses many ethical, legal, practical and psychological dilemmas for doctors. Junior doctors achieve competence in these skills through observation in clinical practice in a traditional apprenticeship model.

Recent data from the Irish Medical Council identifying educational needs of new-entrants to the practice of medicine in Ireland recognises gaps in areas, including communication skills (for patient-centered care, end of life issues and advocacy skills) and legal and ethical aspects of end of life care (autonomy of decision-making, capacity, documentation of care and the legal basis of prescribing). Results of a recent national trainee experience survey in Ireland showed that $74 \%$ of interns did not feel well prepared for the physical and/or emotional demands of clinical practice [1]. 
Dealing with death and dying is a common source of stress and psychological distress to doctors [2]. Stress and poor resilience can lead to syndromes of clinician stress such as burnout, moral distress, compassion fatigue and posttraumatic stress disorder symptoms [3, 4]. Compassion fatigue (also known as secondary posttraumatic stress disorder (PTSD)) is described as diminished emotional energy needed to care for patients and has symptoms similar to primary PTSD, i.e. hyperarousal, avoidance and re-experiencing [3, 5]. PTSD is characterised by exposure to a significant traumatic event and by subsequent development of characteristic symptoms [6]. Secondary PTSD applies to those emotionally affected by the trauma of another [5]. Repeated vicarious exposure to trauma can contribute to compassion fatigue and PTSD symptoms [4]. A recent study reported that one-third of professionals working in a palliative care setting, who were enrolled in mindfulnessbased communication training, reported clinically significant PTSD symptoms [4]. Syndromes of clinician stress can impact on physician mental health, patient care and safety [7-9].

\section{Aims}

- Determine the frequency with which junior doctors deal with end of life care and death.

- Evaluate the impact of patient death on the psychological wellbeing of junior doctors.

\section{Materials and methods}

\section{Research design}

A mixed methods design was adopted which allowed for the triangulation of data between the quantitative and qualitative phases of the study. During the quantitative phase, a questionnaire investigating junior doctors' experience with end of life care and patient death was circulated in two teaching hospitals in Ireland. The qualitative phase involved junior doctors in one of the hospitals participating in critical incident technique (CIT) interviews about their experience of end of life care and patient death.

\section{Quantitative phase}

\section{Questionnaire development}

The questionnaire contained a section on demographics, frequency of carrying out tasks (e.g. notification and certification of death, pronouncing death and documentation, communication with families), desire for knowledge and training and the psychological impact of patient deaths on the study population. During development of the questionnaire, a focus group of researchers (comprising of four doctors) was held to discuss the format and content of the questionnaire. A draft of the questionnaire was piloted before distribution. The final questionnaire was circulated to interns and senior house officers (SHOs) in two university teaching hospitals between September and November 2016. An abbreviated 6-item version of the PTSD Checklist-Civilian version (PCL-C) was incorporated into the questionnaire to assess psychological distress related to the death of a patient (Table 1) [10]. For each of the six questions, the respondents were asked to report on a Likert scale 1 (not at all) to 5 (extremely) as to how much they agreed with the statement yielding a minimum score of 6 and a maximum score of 30 . A score of $\geq 14$ is considered a positive screening test for PTSD scale. The PCL-C yields three subsets which represent symptom clusters of the criteria for posttraumatic stress disorder (PTSD) (Table 1) [11].

Evaluation of the psychometric properties of the PCL-C measure has shown high internal consistency, test-retest reliability, convergent validity and discriminant validity [11]. The PCL-C (6-item short form version) has been found to have high criterion validity when compared with established methods of scoring PTSD such as the Mississippi PTSD scale and the CAPS (Clinician-Administered PTSD scale) [12]. The PCL-C scores highly for diagnostic efficiency $(0.75 \pm 0.06)$, sensitivity $(0.92 \pm 0.19)$ and specificity $(0.72 \pm 0.76)$ [13].

\section{Data collection}

Purposive sampling of junior doctors (interns and SHOs) took place in two teaching hospitals in Ireland. Purposive sampling involves choosing subjects because they possess knowledge, experience or the other attributes relative to the phenomena being explored. The paper-based questionnaire was distributed by a palliative care registrar (HL) at scheduled teaching events in the hospitals. Junior doctors were approached on the wards and asked them to return the completed questionnaire to a single collection point to ensure anonymity. Data was collected between September and November 2016. An information sheet including a distress protocol (in the event that the questionnaire evoked distress in the participant) was provided and written consent was obtained from all participants.

\section{Data analysis}

The survey data was coded and analysed by a statistician (FC) using simple descriptive statistics and independent $t$ tests, to compare junior doctors at different seniorities using SPSS (Statistical Package for the Social Sciences) statistical software. 
Table 1 6-item abbreviated PTSD Checklist-Civilian version (PCL-C with question subsets based on clusters in DSM-IV diagnostic criteria)
Subset: Re-experiencing (cluster B)

Subset: Avoidance (cluster C)

Subset: Hyperarousal (cluster D)
1. Repeated, disturbing memories, thoughts, or images of the incident?

2. Felt very upset when something reminded you of the incident?

3. Avoided activities or situations because they reminded you of this stressful experience?

4. Feeling distant or cut off from other people?

5. Feeling irritable or having angry outbursts?

6. Difficulty concentrating?

\section{Qualitative phase}

\section{Interview design}

Critical incident technique (CIT) interviews were conducted by a registrar in palliative medicine (HL) and by a medical SHO (LM) following training in conducting CIT interviews. Both interviewers work in the hospital where the interviews were conducted and in some cases had an established professional relationship with the interviewees. CIT is a wellestablished qualitative research tool used in many areas of the health sciences used to evaluate behaviour in professional environments. This technique was used to understand the underlying knowledge, skills and attitudes of the respondents through the description of a challenging scenario [14-16], in this case, the death of a patient. The four primary phases to CIT interviews are (i) selection of an appropriate incident, (ii) probing to construct a definitive description of the events surrounding the incident and exploring the reasoning, (iii) investigating team members' rationales for actions taken in relation to the incident and (iv) pinpointing specific root causes of the incident [17]. Opening questions to identify appropriate incidents included statements such as "Can you remember a challenging situation when caring for a patient at end of life?" This was followed by non-directive probing to explore and expand on participant experiences of dealing with death and dying, rationales for actions taken and an exploration of root causes of the incident.

\section{Data collection}

Critical incident technique interviews were conducted by researchers HM, LM with interns, SHOs and registrars in a teaching hospital in Ireland between November 2016 and May 2017. The interviews were carried out between the interviewer and the participant with no other persons present. All participants were given an information sheet which included a distress protocol and signed an informed consent form. Purposive sampling (a preferred method for qualitative studies) [18] was used to select participants with experience of dealing with death and dying patients. To reduce selection bias, a snowballing sampling technique was employed to recruit potential participants. Recruitment continued until new categories, themes or explanations stopped emerging from the data and the research team determined that data saturation had been reached [19]. The sample frame included junior doctors with experience in managing dying patients. The interviews were audio-recorded and transcribed onto a passwordprotected computer. The original audio files were subsequently destroyed. Transcripts were not returned to participants. To maximise the transferability of the research, direct quotes and detailed descriptions were used to clearly represent the data.

\section{Data analysis}

All interviews were audio-recorded using a denaturalised approach and transcribed verbatim by two independent researchers (HL, FC). Investigators independently read all interview transcripts to identify primary themes and subthemes. Data was analysed line-by-line by (FC) without using qualitative data software. The results from the quantitative data served as a provisional starting list of priori codes by which to analyse the data.

An inductive content analysis method was employed to analyse the emerging themes from the qualitative data. Inductive content analysis consists of three main stages: preparation, coding and reporting [20]. This inductive process follows a grounded theory approach in which the categories are informed by the data [21]. Researchers subsequently met to establish a consensus for a coding scheme to inform all later analyses. Any disagreements regarding coding were resolved by discussion to reach consensus. For this research, the researchers developed categories and definitions of common issues that doctors experience during and after the death of a patient which emerged strongly throughout the data. Employing an inductive content analysis allows the researcher to describe the phenomenon of dealing with the death of a patient from the doctors' perspective, to increase understanding and generate knowledge [20]. The formulation of categories requires the researcher, through interpretation, to decide the appropriate categorisation for the data [20]. These definitions were illustrated with examples from the interview data to ensure external heterogeneity and internal homogeneity which were representative of the research findings representing the most prevalent factors influencing doctors' actions when dealing with the death of a patient. 


\section{Results}

\section{Quantitative phase}

\section{Participants}

After excluding missing data resulting from unfinished questionnaires $(n=17)$, our final sample consisted of 110 participants; 39 interns (response rate $44.8 \% ; n=39 / 87$ ) and 71 SHOs (response rate $33.6 \% ; n=71 / 211$ ). The interns had a mean of 3.2 months (range $=4$ months) in clinical practice and the SHOs had 29.2 months (range= 72 months). A total of $81.9 \%(n=86)$ of participants were graduates of Irish medical schools and $18.9 \%(n=20)$ were from medical schools outside of Ireland.

\section{Prevalence of tasks related to end of life}

Table 2 outlines the frequency with which junior doctors carry out tasks associated with end of life care. For SHOs, $90 \%(n=$ $63 / 70$ ) had been involved in a cardiac arrest when the patient has died compared to just $41 \%(n=16 / 39)$ of interns. Chisquare tests indicate that SHOs were significantly more likely, than interns, to have carried out all specified tasks associated with end of life.

\section{Psychological impact of patient death}

A total of $86.4 \%(95 / 110)$ of respondents reported feeling distressed as a result of a patient death, $51.8 \%$ (57/110) at least moderately and $4.5 \%(5 / 110)$ to an extreme level. Of the total sample, $42.7 \%(47 / 110)$ had repeated disturbing memories, $19.1 \%(21 / 110)$ at least moderately and $2.7 \%(3 / 110)$ to an extreme extent. Just under half of the total participants, $48.1 \%$ $(53 / 110)$ were upset by a patient death. $18.2 \%(20 / 110)$ avoided activities that reminded them of the patient death. $24.5 \%(27 / 110)$ admitted to feeling distant or cut off from others. When respondents were asked if they felt angry or described having angry outbursts, $18.2 \%$ (20/110) identified that they had. Some respondents $(28.2 \% ; 31 / 110)$ had experienced difficulty concentrating following a patient death. The results of the breakdown of psychological impact on interns and SHOs are outlined in Tables 3 and 4 respectively.

Of the total sample, 13/110 (11.8\%) of respondents scored positively for PTSD determined by the PCL-C (score greater or equal to 14$)$, consisting of $3 / 39(7.7 \%)$ of interns and $10 / 71$ (14.1\%) SHOs. Using independent sample $t$ tests, no significant difference was found between SHOs and interns on PCLC mean scores $(p=0.117)$.

\section{Education and training around end of life care}

A total of $80 \%(88 / 110)$ respondents expressed a desire for more training in palliative care: when comparing doctors at varying seniorities, this consisted of $71.8 \%$ (28/39) interns and $84.5 \%(60 / 71)$ of SHOs.

\section{Awareness of resources to manage distress}

A total of $60.9 \%(67 / 110)$ respondents are not aware of resources at their disposal should they be feeling distressed/ experiencing low mood, corresponding to $53.8 \%$ (21/39) of interns and $64.8 \%(46 / 71)$ of SHOs.

\section{Qualitative phase}

\section{Participants}

A total of 31 junior doctors were recruited for the qualitative phase of this project: 5 interns, 16 SHOs and 10 registrars. There were 34 separate experiences within these 31 interviews. Response rate was $88.6 \%$ (31/35). Participants that declined reported that their reason for doing so was the lack of an experience to discuss. Of the total sample, $35.3 \%(12 / 34)$ pertained to experiences in intern year, $38.2 \%$ (14/34) to SHO years and $23.5 \%(8 / 34)$ to registrar years. Interviews lasted between 3:08 and 24:39 $\mathrm{min}$. We continued to recruit participants to interview until the researcher determined that data saturation had been reached.

Table 2 Frequency of carrying out tasks related to end of life

\begin{tabular}{|c|c|c|c|c|}
\hline End of life tasks (at least once) & Interns $(\%)$ & SHOs & $\begin{array}{l}\chi^{2} \text { sig. difference between } \\
\text { interns and SHOs }\end{array}$ & Total \\
\hline Pronouncing death & $24 / 39(61.5 \%)$ & $66 / 71(93 \%)$ & 0.000 & $90 / 110(81.8 \%)$ \\
\hline $\begin{array}{l}\text { Involved in a cardiac/respiratory arrest when } \\
\text { the patient has died }\end{array}$ & $16 / 39(41 \%)$ & $63 / 70(90 \%)$ & 0.000 & $79 / 109(72.5 \%)$ \\
\hline Discuss patient at end of life with relatives & $25 / 39(64.1 \%)$ & $58 / 71(81.7 \%)$ & 0.002 & $83 / 110(75.5 \%)$ \\
\hline Discuss resuscitation with relatives & $9 / 39(23.1 \%)$ & $51 / 71(71.8 \%)$ & 0.000 & $60 / 110(54.5 \%)$ \\
\hline Discuss withdrawal of life-sustaining measure & $7 / 39(17.9 \%)$ & $30 / 71(42.3 \%)$ & 0.010 & $37 / 110(33.6 \%)$ \\
\hline Notified coroner & $4 / 39(10.3 \%)$ & $19 / 71(26.8 \%)$ & 0.042 & $23 / 110(20.9 \%)$ \\
\hline
\end{tabular}


Table 3 Psychological impact after experiencing patient death: intern data

\begin{tabular}{llll}
\hline & Total & $\begin{array}{l}\text { At least moderately (score greater } \\
\text { than or equal to 3 on Likert) }\end{array}$ & $\begin{array}{l}\text { Extremely } \\
\text { (score 5 on Likert) }\end{array}$ \\
\hline Distressed & $31 / 39(79.5 \%)$ & $19 / 39(48.7 \%)$ & $1 / 39(2.6 \%)$ \\
Repeated disturbing memories & $12 / 39(30.8 \%)$ & $5 / 39(12.8 \%)$ & $1 / 39(2.6 \%)$ \\
Upset & $12 / 39(30.8 \%)$ & $4 / 39(10.3 \%)$ & $0 / 39(0 \%)$ \\
Avoided activities that reminded them of stressful experience & $4 / 39(10.3 \%)$ & $3 / 39(7.7 \%)$ & $0 / 39(0 \%)$ \\
Felt distant or cut off from others & $7 / 39(17.9 \%)$ & $2 / 39(5.1 \%)$ & $0 / 39(0 \%)$ \\
Feeling angry or had angry outbursts & $4 / 39(10.3 \%)$ & $2 / 39(5.1 \%)$ & $1 / 39(2.6 \%)$ \\
Difficulty concentrating & $9 / 39(23.1 \%)$ & $3 / 39(7.7 \%)$ & $0 / 39(0 \%)$ \\
\hline
\end{tabular}

Qualitative interviews explored issues experienced by doctors when caring for patients at end of life. Four primary themes emerged from the data: (i) difficulty with communicating death and dying to patients and family members, (ii) lack of awareness of support structures for doctors in the aftermath of patient death, (iii) lack of knowledge and understanding of the legal processes surrounding patient death including post-mortem, coroner, advanced care directives and (iv) lack of preparation for the impact of patient death and dying.

\section{(i) Communicating death to family members}

The most common issue identified by doctors when delivering end of life care and interacting with patient death was difficulty in communicating death to family members, involved in 59\% (20/34) of scenarios. Junior doctors expressed discomfort in delivering bad news to families if they have inadequate information to deliver to families regarding the patient's treatment. The data suggested that there can be a lack of support from senior clinicians and that there is delayed decision-making with regard to care goals.

"I actually found it quite difficult. I was an intern and had little experience so I found it quite difficult because her family would stop you in the corridor looking for answers for different things and I didn't necessarily have them so I would often have to just defer and wait until somebody else came along to answer them. A lot of it I wasn't able to answer for them." [Intern 5]

\section{(ii) Support structures}

A second issue identified was the lack of support structures, identified by $17 / 34(50 \%)$ of participants. The ability to deliver bad news and develop a positive emotional and professional strategy to manage the process of patient death comes with experience. Participants admitted that they adopted a strategy of distancing themselves from their patients in order to mediate the distress of patient deterioration and eventual death. Doctors perceive a lack of support structures or formal professional service to reflect on patient death.

"I found it hard to have those heavy conversations with the patient- I felt somewhat burdened going home in the evenings ... It has had a psychological impact and it affects my decisions to this day ... I hope that as a result of this case that I would be more assertive when talking to my seniors about my concerns. A lot of learning will come with experience. I think that confidence comes with experience." [SHO 3]

Table 4 Psychological impact after experiencing patient death: SHO data

\begin{tabular}{llll}
\hline & Total & $\begin{array}{l}\text { At least moderately (score greater } \\
\text { than or equal to 3 on Likert) }\end{array}$ & $\begin{array}{l}\text { Extremely } \\
\text { (score 5 on Likert) }\end{array}$ \\
\hline Distressed & $64 / 71(90.1 \%)$ & $38 / 71(53.5 \%)$ & $4 / 71(5.6 \%)$ \\
Repeated disturbing memories & $35 / 71(49.3 \%)$ & $16 / 71(22.5 \%)$ & $2 / 71(2.8 \%)$ \\
Upset & $41 / 71(57.7 \%)$ & $17 / 71(23.9 \%)$ & $0 / 71(0 \%)$ \\
Avoided activities that reminded them of stressful experience & $16 / 71(22.5 \%)$ & $4 / 71(5.6 \%)$ & $0 / 71(0 \%)$ \\
Felt distant or cut off from others & $20 / 71(28.2 \%)$ & $6 / 71(8.5 \%)$ & $1 / 71(1.4 \%)$ \\
Feeling angry or had angry outbursts & $16 / 71(22.5 \%)$ & $6 / 71(8.5 \%)$ & $1 / 71(1.4 \%)$ \\
Difficulty concentrating & $22 / 71(31 \%)$ & $4 / 71(5.6 \%)$ & $0 / 71(0 \%)$ \\
\hline
\end{tabular}




\section{(iii) Legal process of death}

Lack of knowledge of the systems and the process of patient death was involved in 13/34 interviews (38\%). The participants reported feeling totally unprepared when asked to contact the coroner, recommend a patient post-mortem or be called to an inquest into the death of a patient. There was a lack of clarity as to the role of the junior doctor in these scenarios and what was appropriate for them to be doing.

"To me the issues in this case were the timing of withdrawal of active treatment, the family's role in this decision and how to proceed once active treatment is stopped like should one continue parenteral fluids? I felt unsure about a lot of these issues" [SHO 1]

and

"The reg (istrar) on that night said that we will have to clarify with the coroner and notify the coroner and I almost felt like I was being asked to do it which I was definitely not comfortable with as I was only a few weeks on the job." [SHO 10]

\section{(iv) Emotional impact of patient death}

The lack of preparedness for impact of patient death and dying on the doctor was identified in 14/34 (41\%) of interviews. The medical compulsion to treat actively as instructed by medical training appears to accentuate the distress felt by doctors when a patient dies under their care. Participants agreed that when a patient was transferred to palliative care, it seemed to indicate a failure on the part of the doctor to effectively treat the patient.

"I think it's probably different now but certainly when I was in medical school it wasn't taught well I think and there was still a sense that palliative meant maybe a failure... that you failed to cure ... Now I don't know how you teach that. I think we're a little bit better about teaching that now." [Registrar 6]

\section{Discussion}

\section{Main findings}

Junior doctors are frequently carrying out tasks related to end of life care. This poses many ethical, legal, practical and psychological dilemmas for an underprepared doctor. Our study has identified specific areas in which junior doctors are experiencing difficulties when caring for patients at end of life.

They are potentially vulnerable to psychological distress due to lack of skills and knowledge around end of life care, lack of support from colleagues and a lack of timely decisionmaking regarding goals of care. There is also a lack of awareness around role and responsibilities.

\section{What this study adds}

This study has identified a number of learning needs for doctors. Educational interventions at undergraduate and postgraduate level are required to better prepare junior doctors for the practical challenges of end of life care and death. Suggested interventions include simulated training in breaking bad news, the discussion around withdrawal of active care and in practical tasks such as completing relevant paperwork and training for doctors on the indications for post-mortem and coroner referral.

This study has also highlighted a perceived lack of structured support in the aftermath of patient death and a perception by some doctors that a patient death indicates a failure on the part of the doctor. Increased awareness of the vulnerability of junior doctors and the importance of identifying those in extreme distress is essential. To address this, it is important to put systems in place to mentor and support colleagues and team members.

In addition to educational interventions, a change in the current anti-emotion culture in medicine is required which would facilitate discussion and encourage those in distress to seek help.

\section{Limitations of the study}

The sample size for the questionnaire was small which may limit generalisability. The numbers of interns and SHOs were unequal; however, this was a proportional representation of the total interns and SHOs in the hospitals sampled. Approaching participants directly may have contributed to selection bias. In order to account for selection bias, the quantitative and qualitative data was anonymised and participants were recruited from two discrete sites. The PCL-C is indicative of self-reported symptoms rather than using an objective assessment of PTSD, and therefore the results are not diagnostic of PTSD. There is no normative data on the levels of psychological distress in junior doctors as a result of patient death to draw a comparison.

Funding This study was funded by the National Doctors Training and Planning, Health Service Executive.

\section{Compliance with ethical standards}

Conflict of interest The authors declare that they have no conflict of interest.

Ethical consideration Ethical approval was obtained from the Clinical Research Ethics Committee, Galway University Hospitals. Chairperson: Professor Gerard Loftus. Approval on 14/9/16 (Reference 1589). Written informed consent was provided by all participants. 


\section{References}

1. Irish Medical Council. Your training counts trainee. Experiences of clinical learning environments in Ireland. Available from https:// www.medicalcouncil.ie/News-and-Publications/Reports/YourTraining-Counts-2015-pdf-.pdf (Accessed 24th May 2015)

2. Firth-Cozens J, Morrison LA (1989 Apr 1) Sources of stress and ways of coping in junior house officers. Stress Med 5(2):121-126

3. Sanchez-Reilly S, Morrison L, Carey E, Bernacki R, O’Neill L, Kapo J, Periyakoil V, Thomas J (2013) Caring for oneself to care for others: physicians and their self-care. J Support Oncol 11(2):75-81

4. O'Mahony S, Gerhart J, Grosse J et al (2015) Posttraumatic stress symptoms in palliative care professionals seeking mindfulness training: prevalence and vulnerability. Palliat Med 30(2):189-192

5. Kearney M, Weininger R, Vachon M et al (2009) Self-care of physicians caring for patients at the end of life. JAMA 301(11):1155

6. Conn P (2016) Conn's translational neuroscience. Press, Academic

7. Shanafelt $\mathrm{T}$ (2002) Burnout and self-reported patient care in an internal medicine residency program. Ann Intern Med 136(5):358

8. West C, Huschka M, Novotny P et al (2006) Association of perceived medical errors with resident distress and empathy. JAMA 296(9):1071

9. O'Connor P, Lydon S, O'Dea A, Hehir L, Offiah G, Vellinga A, Byrne D (2017) A longitudinal and multi-centre study of burnout and error in Irish junior doctors. Postgrad Med J 93:660-664

10. Weathers FW, Litz BT, Herman D et al (1994) The PTSD checklistcivilian version (PCL-C). Boston, MA, National Center for PTSD
11. Bell C (1994) DSM-IV: diagnostic and statistical manual of mental disorders. JAMA 272(10):828

12. Lang A, Stein M (2005) An abbreviated PTSD checklist for use as a screening instrument in primary care. Behav Res Ther 43(5):585-594

13. O'Dea A, O'Connor P, Keogh I (2014) A meta-analysis of the effectiveness of crew resource management training in acute care domains. Postgrad Med J 90(1070):699-708

14. Flanagan JC (1954) The critical incident technique. Psychol Bull 51(4):327-358

15. Fitzgerald K, Seale NS, Kerins CA et al (2008) The critical incident technique: a useful tool for conducting qualitative research. J Dent Educ 72(3):299-304

16. Bradley CP (1992 Mar 1) Turning anecdotes into data - the critical incident technique. Fam Pract 9(1):98-103

17. O'Connor P, O'Dea A, Melton J (2007) A methodology for identifying human error in US Navy diving accidents. Hum Factors 49(2):214-226

18. Smith J, Flowers P, Larkin M (2009) Interpretative phenomenological analysis: theory, method and research. Sage

19. Guest G, Bunce A, Johnson L (2006) How many interviews are enough? An experiment with data saturation and variability. Field methods 18(1):59-82

20. Elo S, Kyngäs H (2008) The qualitative content analysis process. J Adv Nurs 62(1):107-115

21. FitzGerald K, Sue S, Kerins C, McElvaney R (2008) The critical incident technique: a useful tool for conducting qualitative research. J Dent Educ 72(3):299-304 